\title{
2.5 D Facial Analysis via Bio-Inspired Active Appearance Model and Support Vector Machine for Forensic Application
}

\author{
Siti Norul Huda Sheikh Abdullah \\ Siti Norul Huda Sheikh Abdullah
Faculty of Information Science and \\ Technology, \\ Universiti Kebangsaan Malaysia, \\ Bandar Baru Bangi, Malaysia
}

\author{
Mohammed Hasan Abdulameer \\ Department of Computer Science, \\ Faculty of Education for Women, \\ University of Kufa, \\ Iraq
}

\author{
Nazri Ahmad Zamani \\ Digital Forensics lab, \\ CyberSecurity Malaysia, \\ Seri Kembangan, \\ Malaysia
}

Fasly Rahim

Faculty of Science and Technology

Universiti Kebangsaan Malaysia,

Bandar Baru Bangi,

Malaysia

\author{
Mohd Zakree Ahmad Nazri \\ Faculty of Information Science and Technology, \\ Universiti Kebangsaan Malaysia, \\ Bandar Baru Bangi, Malaysia
}

\author{
Khairul Akram Zainol Ariffin \\ Faculty of Information Science and \\ Technology, \\ Universiti Kebangsaan Malaysia, \\ Bandar Baru Bangi, Malaysia
}

\author{
Zulaiha Othman \\ Faculty of Information Science and \\ Technology, \\ Universiti Kebangsaan Malaysia, \\ Bandar Baru Bangi, Malaysia
}

\begin{abstract}
In this paper, a fully automatic 2.5D facial technique for forensic applications is presented. Feature extraction and classification are fundamental processes in any face identification technique. Two methods for feature extraction and classification are proposed in this paper subsequently. Active Appearance Model (AAM) is one of the familiar feature extraction methods but it has weaknesses in its fitting process. Artificial bee colony (ABC) is a fitting solution due to its fast search ability. However, it has drawback in its neighborhood search. On the other hand, PSO-SVM is one of the most recent classification approaches. However, its performance is weakened by the usage of random values for calculating velocity. To solve the problems, this research is conducted in three phases as follows: the first phase is to propose Maximum Resource Neighborhood Search (MRNS) which is an enhanced ABC algorithm to improve the fitting process in current AAM. Then, Adaptively Accelerated PSO-SVM (AAPSO-SVM) classification technique is proposed, by which the selection of the acceleration coefficient values is done using particle fitness values in finding the optimal parameters of SVM. The proposed methods AAMMRNS, AAPSO-SVM and the whole 2.5D facial technique are evaluated by comparing them with the other methods using new 2.5D face image data set. Further, a sample of Malaysian criminal real case of CCTV facial investigation suspect has been tested in the proposed technique. Results from the experiment shows that the proposed techniques outperformed the conventional techniques. Furthermore, the 2.5D facial technique is able to recognize a sample of Malaysian criminal case called "Tepuk Bahu" using CCTV facial investigation.
\end{abstract}

Keywords-Face recognition; active appearance model; ant bee colony; particle swarm optimization; support vector machine

\section{INTRODUCTION}

The reliability of evidence in a court of law is dependent upon how the evidence is handled, how it is interpreted, and how it is presented. The fundamental principle behind digital forensics is maintaining integrity and provenance of media upon seizure, and throughout the analysis and handling process [1]. The evidence has to undergo a number of processes to ensure its integrity and admissibility into the court of law. In the past, a number of models have been considered as the best practices for conducting digital forensics and the common processes in those models are evidence identification, analysis and interpretation of the result and finally presentation of the findings [2]. In forensics, evidence can be explained with Locard's Exchange Principle, which holds that the perpetrator of a crime will bring something into the crime scene and leave something from it [3]. This principle is the foundation of forensic sciences and this extends to the digital forensics, and video forensic is no exception. The role of forensic science is to uncover the traces left behind from a crime scene, whether it is physically, chemically or digitally.

Facial recognition is the application of biometrics [4], and in video exhibit, it is an essential forensic analysis in order to improve the clarity of the face for a human observer. The facial clarified is said to be important, as it will be used to assist law enforcement agency in their investigation, litigation or legal process. The challenges faced by this particular discipline of forensics contributed mostly by the quality of the exhibit itself. For example, surveillance video from CCTV 
system is always backed up with down-sampled resolution. The surveillance video stored inside the CCTV's Digital Video Recorder (DVR) is normally kept at resolution 720 pixels wide with H.264 compression. After a certain period of time, the video resolution is down-sampled at lower size to accommodate more space for the recording. The qualities of the recording were found to be low with signal noise, color noise, compression artefacts, illumination problems and many kinds of blurring. Further, the people are usually walking free, and it is impossible to always keep faces frontal to the cameras. Hence, face images captured by the CCTV are nonideal due to many factors: pose, illumination, expression, distance and weather [5]. These degradations have caused many dead ends to video forensic analysis. Consequently, the forensic investigation reports were challenged or rejected by court due to inefficient evidence presented by the reports.

The paper highlights on the methodology of 2.5 D Facial Analysis via Bio-Inspired Active Appearance Model and Support Vector Machine. It is organized as follows: In Section 2, the discussion on the application of face recognition in digital forensics will be outlined. In Section 3, the state of the art for face recognition is highlighted. Then it continues with the proposed model in Section 4 and the result is discussed in Section 5. Finally, the overall work of this paper is summarized in the last section.

\section{APPLICATION OF FACE RECOGNITION IN DigITAL FORENSICS}

Forensics facial identification system is a biometric system that analyses face-matching result for investigation or for litigation process. In other variation, this can also be defined as a face recognition system for the forensics purposes. As the term identification is being used, therefore it emphasizes the 1: $N$ matching.

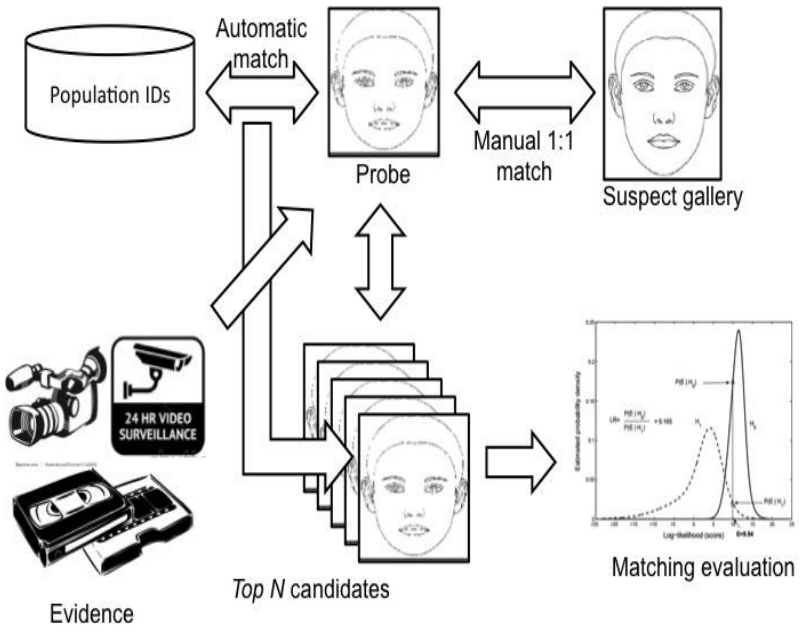

Fig. 1. The paradigm of forensics face recognition system

The aim of the analysis is to find the top $N$ matches for a probe, in which is extracted from a photo or video exhibit. For the database of IDs, two types of IDs are prepared-1) the population face enrolment IDs and 2) a gallery of suspect IDs face photos. The matches will look into the top $N$ matches result and see if the suspect IDs comes up at the top or not.
The analysis would also examine on the matching accuracy and the numbers of false matches. The overall work flow for face recognition is shown in Fig. 1.

There are four applications of facial identification in digital forensics such as:

- Criminal justice: The facial identification system is used to ensure that the database does not contain multiple records for a single individual.

- Identity checks in the field: To get automated decisions without requiring expert analysis.

- Criminal investigation and information: Analysing surveillance videos, witness' camera, Internet sites or smartphones.

- Prevention: Background check to prevent people with criminal history. The technology can also be used for passport control's watch list.

\section{STATE OF THE ART}

From the point of forensic analysis view: The first attempt of face recognition by humans was reported in a British court in 1871 where the suspect was identified by comparing a pair of facial photographs [6]. Then, the forensic tool for face recognition is introduced by Bertillon's work where in the practice, two photographs of a subject are captured, one from the front and one in profile. The same approach is still being used in law enforcement's mug shots [7]. The application of face recognition for forensic is at an advantage if the evidence is presented - whether it is a still image or a video. The image and video can be from both analogue and digital camera, provided the original storage or the negative are also seized. Furthermore, face recognition is a good modality for forensic as the biometric is not intrusive and non-contact [8]. Another advantage of face modality in forensic is that the evidence and the reference that contain the modality is at abundance. Face image is already in existence in any country's civil and criminal files. In addition to mug shots, face images are recorded and digitized for citizens' identity, licenses, passport and visas [9]-[11]. There is also a potential wealth of facial images recorded by alert observers using smartphones, and ever growing number of websites, blogs and social medias. Just like the old Bertillon's system, face recognition is used to establish the identity of the person inside, as evidence to identity which is a reference, for example, inside a surveillance video footage of a robbery. The video can be tended to court as primary evidence against apprehended suspects. To verify the men inside the video footage is the men apprehended face recognition is used.

From the point of image processing view: In verifying the evidence in the forensic work related to image and CCTV, the digital forensic analysts perform manual operations to match facial images with huge databases of mug-shots, which are most challenging and consumes huge time [12]. Nevertheless, a number of studies have focused on 2D and 3D face recognition. However, using 2D images for face recognition is sensitive to various factors, such as, illumination, pose variations, and facial expressions [5] [13]. On the other hand, using 3D image has been adversely 
impacted by changes in facial expressions [14]. Furthermore, 3D techniques also encounter few other restrictions, including, costly gadgets, and computational complication [15]. In contrast, earlier studies have highlighted that 2.5D face image is capable to address the limitations of using 2D and 3D face image recognition [16]-[18]. One of the essential processes in face recognition is the feature extraction. Previously, the Active Appearance Model (AAM) is frequently used for the facial extraction. Although it is a popular method for the facial identification, this model has weaknesses in terms of fitting process [19].

From the perspective of Bio-inspired machine learning algorithms: Artificial Bee Colony $(\mathrm{ABC})$ is an effective fitting solution and has been extensively used in many fields, owing to its rapid search potential [20]. The ABC has also been effectively applied to address challenging optimization problems [21]. However, this approach has a setback in terms of their neighborhood search, where it randomly generates new food sources. Classification is another crucial part in any face recognition technique. Previously, most of classification techniques use Particle Swam Optimization with Support Vector Machine (PSO-SVM) [22] which employs PSO to find the optimal parameters for SVM, even though its performance has deteriorated by the usage of random values for calculating velocity.

\section{PROPOSED MODEL}

The proposed face recognition technique is performed in two main phases, feature extraction and classification. The feature extraction stage uses the proposed active appearance model approach based on Maximum Resource Neighborhood Search (MRNS) which is an enhanced ABC algorithm (AAMMNRS), and the classification stage is based on the proposed AAPSO-SVM [23]. These two phases are performed repeatedly on the input database face images, and thus the face images are recognized more effectively. The basic structure of the proposed 2.5D facial technique is shown in Fig. 2.

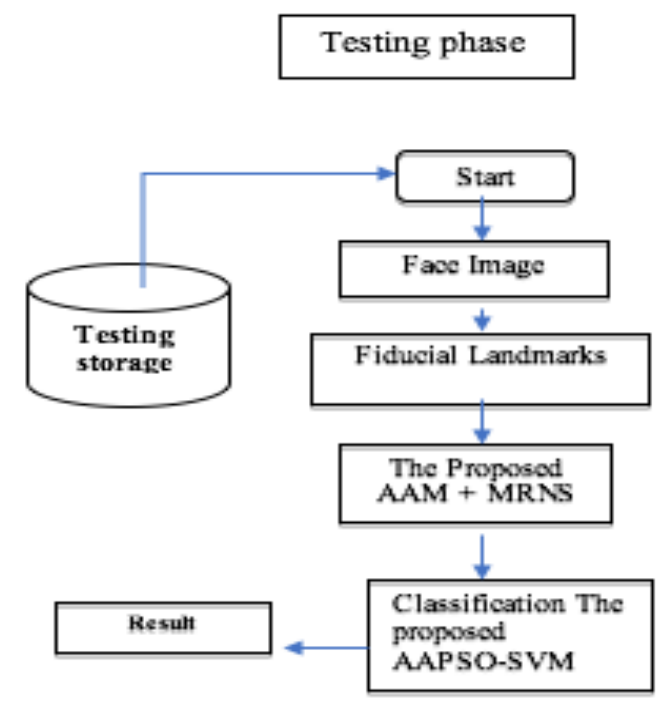

(A)

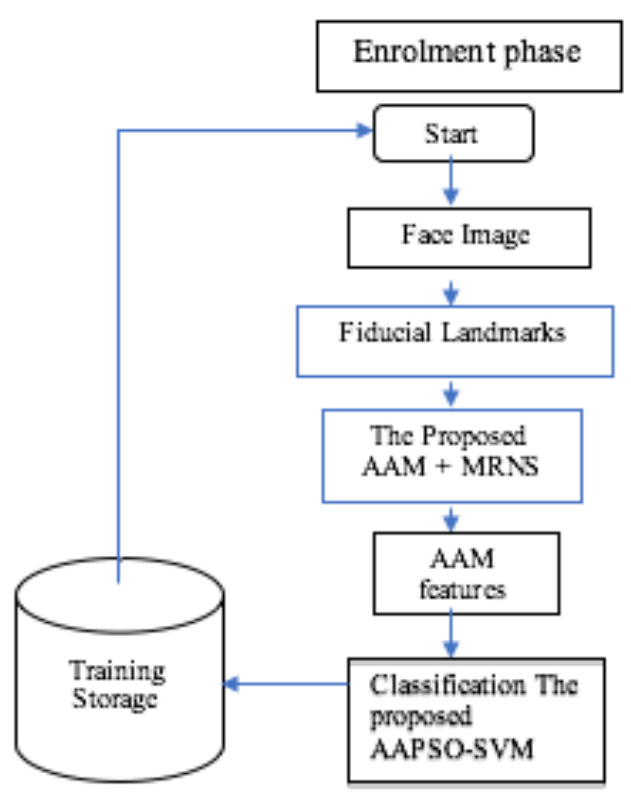

(B)

Fig. 2. The main components of the proposed 2.5D facial forensic technique; (A) for training, (B) for enrolment.

The proposed feature extraction technique utilizes AAM for extracting the shape and appearance features from the database images. In addition, it is crucial to have appropriate fitting for extracting AAM based features. Consequently, the fitting performance has been enhanced by presenting a new maximum resource neighborhood search (MNRS) algorithm, where the searching performance has been accelerated by considering the quality of the best food source. Depending on the quality of best food source, the new food sources are generated by the neighborhood search of the algorithm. The proposed feature extraction method is mainly comprised of 1) Feature Extraction using AAM Modeling; 2) the proposed MRNS Algorithm; 3) fitting using MRNS algorithm.

The standard PSO method has been utilized in many research works to obtain optimal problem solutions. The major drawback of the PSO is the random value selection during new particle generation; that is, in the velocity computation, the acceleration coefficients are generated randomly. The random value selection in the velocity process means that the generated particles will also be random. Random populations do not produce more accurate results. Hence, to acquire a more accurate result and to reduce this PSO drawback, adaptive acceleration particle swarm optimization (AAPSO) is proposed. In order to obtain more accurate classification results, the SVM parameters will be optimized by our AAPSO. The utilization of AAPSO in the SVM parameter optimization will reduce the PSO drawback and improve the classification result accuracy.

\section{EXPERIMENTAL RESULT}

This section presented the experimental results and performance of the proposed 2.5D facial forensic technique 
which depends on the proposed AAM-MRNS and AAPSOSVM methods. The performances have been evaluated based on the fitting error in feature extraction and accuracy rate of the whole classification process in this technique. The experiment has been conducted using the property $2.5 \mathrm{D}$ face dataset and a sample of Malaysian real case of CCTV facial investigation. Two evaluation steps have been used to analyze the final 2.5D facial forensic technique. The first step has evaluated the proposed 2.5D technique using fitting errors based on the performance of the proposed AAM-MRNS method; while the second step has evaluated the $2.5 \mathrm{D}$ technique using recognition accuracy rate based on the performance of proposed AAPSO-SVM method.

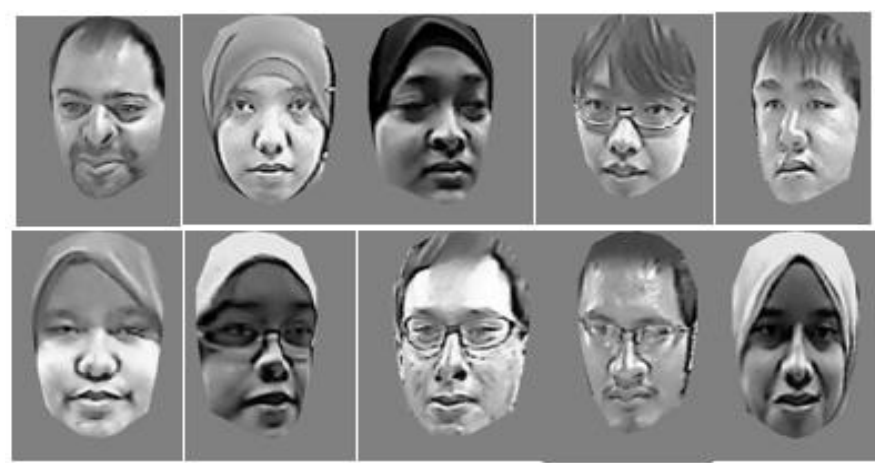

Fig. 3. Ten sample images from 2.5D face dataset prepared by UKMCybersecurity Digital Forensic Lab.

The performance of the proposed 2.5D technique has been evaluated by UKM property dataset namely $2.5 \mathrm{D}$ face images dataset. This dataset has been divided to training and testing images. 18 images have been utilized for training and 6 images have been used for testing. The performance of the proposed technique has been analyzed and compared to the conventional methods like, conventional AAM and Adaptive AAM [24]. The samples of 2.5D images utilized in the recognition process are shown in Fig. 3.

During the analysis of the performance, 10 rounds of experiments have been conducted with the $2.5 \mathrm{D}$ face and iris images. Table 1 and Fig. 4 illustrate the results of fitting error values for the proposed and conventional techniques, acquired in 10 rounds of experiments. Based on the result in Table 1 and Fig. 4 the proposed $2.5 \mathrm{D}$ technique has achieved minimum fitting error as average as compared to the conventional AAM and adaptive AAM. The results reflect the stability in different datasets not only 2D datasets.

\section{Fitting Error versus Methods}

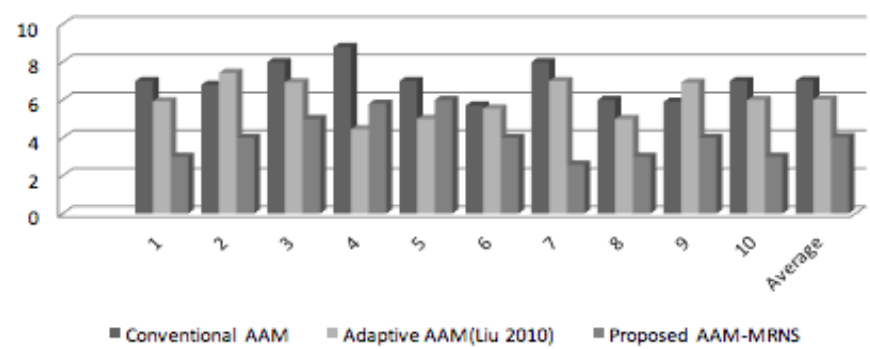

Fig. 4. Fitting error values of the proposed AAM-MRNS and conventional AAM techniques.
TABLE. I. FITTING ERROR FOR PROPOSED AND CONVENTIONAL RECOGNITION TECHNIQUES ON FACIAL DATASET

\begin{tabular}{|l|l|l|l|}
\hline \multirow{2}{*}{ Exp } & \multicolumn{2}{|l|}{ Fitting Error } & \multicolumn{2}{|l|}{} \\
\cline { 2 - 4 } & Conventional AAM & $\begin{array}{l}\text { Adaptive AAM } \\
{[24]}\end{array}$ & $\begin{array}{l}\text { Proposed AAM- } \\
\text { MRNS }\end{array}$ \\
\hline 1 & 7 & 5.93 & 3 \\
\hline 2 & 6.8 & 7.43 & 4 \\
\hline 3 & 8 & 6.93 & 5 \\
\hline 4 & 8.8 & 4.45 & 5.8 \\
\hline 5 & 7 & 5 & 6 \\
\hline 6 & 5.7 & 5.54 & 4 \\
\hline 7 & 8 & 7 & 2.58 \\
\hline 8 & 6 & 5 & 3 \\
\hline 9 & 5.9 & 6.92 & 4 \\
\hline 10 & 7 & 6 & 3 \\
\hline Average & 7.02 & 6.02 & $\mathbf{4 . 0 3 8}$ \\
\hline
\end{tabular}

In the accurate rate evaluation, the performance of proposed $2.5 \mathrm{D}$ technique has been evaluated by the accuracy rate based on AAM.MRNS+AAPSO.SVM. The recognition performance has been analyzed and compared with AAM $\mathrm{ABC}+$ OPSO.SVM, AAM.MRNS + OPSO-SVM, AAM.ABC + AAPSO-SVM methods, and with PSO-SVM which is one of the most recently developed face recognition approaches. Table 2 and Fig. 5 illustrate the accuracy of face images recognition of the proposed technique and others.

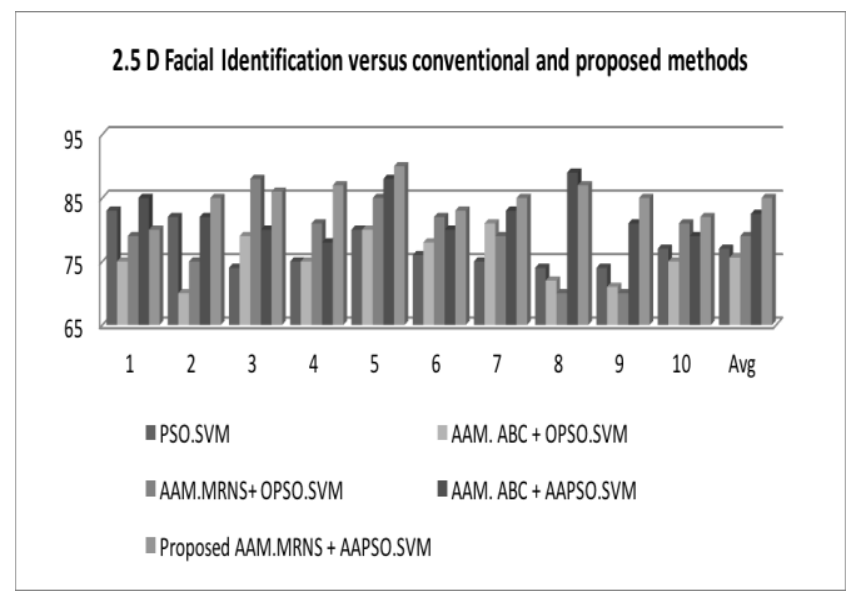

Fig. 5. Accuracy performance of the proposed 2.5 technique against the other techniques.

Based on the Table 2 and Fig. 4, it is evident that the proposed 2.5D technique that used AAM-MRNS for feature extraction and AAPSO-SVM for recognition, has given higher face image recognition accuracy, than the other techniques.

The high-performance results show that, the proposed 2.5D face identification technique is capable of more accurately recognizing the face images. 
TABLE. II. PERFORMANCE OF PROPOSED 2.5D TeChNIQUES AND OTHER RECOGNITION TECHNIQUES FOR FACE DATASET.

\begin{tabular}{|c|c|c|c|c|c|}
\hline \multirow[b]{2}{*}{$\operatorname{Exp}$} & \multicolumn{5}{|c|}{ Accuracy (\%) } \\
\hline & PSO.SVM & $\begin{array}{l}\text { AAM.ABC } \\
+ \\
\text { OPSO.SV } \\
M\end{array}$ & $\begin{array}{l}\text { AAM.M } \\
\text { RNS + } \\
\text { OPSO.S } \\
\text { VM } \\
\end{array}$ & $\begin{array}{l}\text { AAM.A } \\
\text { BC }+ \\
\text { AAPSO. } \\
\text { SVM } \\
\end{array}$ & $\begin{array}{l}\text { Proposed } \\
\text { AAM.MRNS } \\
+ \\
\text { AAPSO.SVM }\end{array}$ \\
\hline 1 & 83 & 75 & 75 & 85 & 80 \\
\hline 2 & 82 & 70 & 75 & 82 & 85 \\
\hline 3 & 74 & 79 & 88 & 80 & 86 \\
\hline 4 & 75 & 75 & 81 & 78 & 87 \\
\hline 5 & 80 & 80 & 85 & 88 & 90 \\
\hline 6 & 76 & 78 & 82 & 80 & 83 \\
\hline 7 & 75 & 81 & 79 & 83 & 85 \\
\hline 8 & 74 & 72 & 70 & 89 & 87 \\
\hline 9 & 74 & 71 & 70 & 81 & 85 \\
\hline 10 & 77 & 75 & 81 & 79 & 82 \\
\hline Ave & $77 \%$ & $75.6 \%$ & $79 \%$ & $82.5 \%$ & $85 \%$ \\
\hline
\end{tabular}

In addition, a sample of Malaysian real case of CCTV facial investigation has been tested on the proposed $2.5 \mathrm{D}$ technique. Several images taken from camera at different crime scene but related to single stealing case namely "Tepuk bahu" case had been observed to be matched. The suspects (Fig. 6) conducted a serial of stealing crimes at various ATM banks in different states in Malaysia. Three Bolivian suspects conducted the crime by setting a plot with one person observing the pin number of a victim, then another person would steal the victim's ATM card by bowing after clapping the victim's shoulder and informing her of her fallen money on the floor. Another suspect would then withdraw the money using stolen ATM card after receiving all details. The source video image has been extracted to tiff image with $480 \times$ 320 image size. We cropped all suspects' face, resizing them and evaluated by using cross validation approach. We conducted two evaluations based on fitting error and precision recall. The result showed that the proposed $2.5 \mathrm{D}$ technique was able to recognize the suspect from CCTV sample.

\section{CONCLUSION}

In this paper, a fully automatic $2.5 \mathrm{D}$ facial technique for forensic applications had demonstrated its performance on the new property $2.5 \mathrm{D}$ data set as well as Malaysian real case of CCTV facial investigation sample.

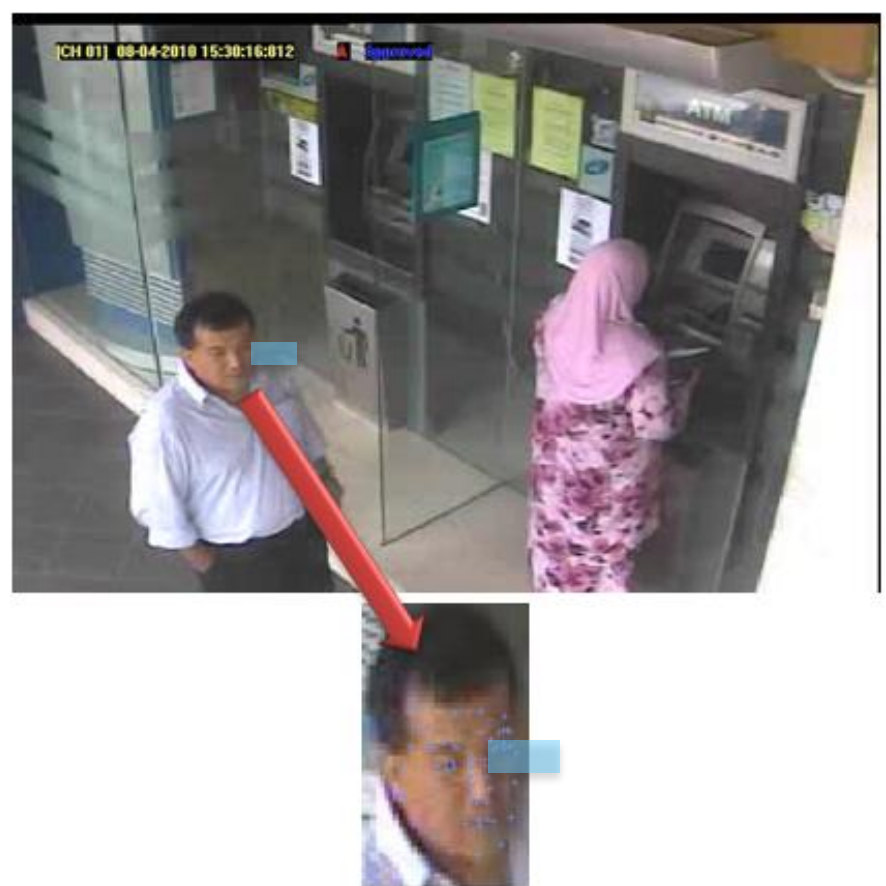

Fig. 6. Examples of (above) life incident dated 8 April 2010 timed 15:30 and (below) extracted face images with annotated landmarks Malaysian real case from CCTV.

A number of contributions were presented while addressing major problems in the area of face recognition. Our contributions include: a modified feature extraction method using AAM based on new algorithm called MRNS (AAMMRNS), classification method named as Adaptively Accelerated Particle Swarm Optimization based on SVM (AAPSO-SVM) and fully automatic 2.5D facial technique based on AAM-MRNS and AAPSO-SVM methods.

In the experimental results, the comparisons were done with the conventional AAM and adaptive AAM in feature extraction part; and with the PSO-SVM as well as the other techniques for the recognition part. The average error rate for the proposed 2.5D technique based on (AAM-MRNS) on the $2.5 \mathrm{D}$ face dataset was $4.038 \%$ and $7.02 \%, 6.02 \%$ for conventional AAM and adaptive AAM respectively. On the other hand, the accuracy rate was used to evaluate the recognition performance for the final proposed $2.5 \mathrm{D}$ technique. In the recognition stage, the proposed 2.5D technique (AAM.MRNS+AAPSO.SVM) technique was compared with (AAM.ABC+ OPSO.SVM), (AAM.MRNS + OPSO.SVM), (AAM.ABC + AAPSO.SVM), and with PSOSVM methods. The average accuracy rate for the proposed 2.5D technique on $2.5 \mathrm{D}$ face images were $85 \%$ and $77 \%$ for PSO-SVM; while 75.6, 79, 82.5 for (AAM.ABC+ OPSO.SVM), (AAM.MRNS + OPSO.SVM), (AAM.ABC + AAPSO.SVM) correspondingly. Furthermore, the 2.5D technique was able to recognize the suspect of Malaysian real case of CCTV investigation.

For the future work, the research will look into several fields to ensure the methodology can assist in helping the 
current video forensics problem. Further, the research is currently exploring in the Graphics Processing Unit (GPU) to enhance and optimize the algorithm performance.

\section{ACKNOWLEDGMENT}

This research was initially funded by the Ministry of Education through ERGS/1/20 IISTG/UKM/2/48 (TK) under the title of 2D-3DHybrid Face Matching via Fuzzy Bees Algorithm for Forensic Identification, Universiti Kebangsaan Grant DIP 2015-023 and FRGS/2/2014/ICT02/UKM/02/1 entitled Crime Prediction Using Spatio-Temporal Pattern with Rough Fuzzy Sets Based Ensemble Learning”.

\section{REFERENCES}

[1] Daniel B. Garrie, Digital Forensic Evidence in the Courtroom: Understanding Content and Quality, Northwestern Journal of Technology and Intellectual Property, Vol 12, Issue 2, 2014.

[2] M. D. Kohn, M.M Eloff, J.H.P Eloff, Integrated Digital Forensic Process Model, Computer \& Security, Vol 38, pp. 103-115, 2013.

[3] Anil K. Jain, Arun Ross, "Bridging the Gap: from Biometrics to Forensics", Philosophical Tractions of The Royal Society, 2015.

[4] Dong Yi, Zhen Lei, Stan Z. Li, Towards Pose Robust Face Recognition, IEEE Conference on Computer Vision and Pattern Recognition (CVPR), pp. 3539-3545, 2013.

[5] Rahul D. Dhotkar, Prakash R. Chandore, Prashant N. Chatur, "Face Recognition techniques and its application", International Journal of Application in Engineering \& Management (IJAIEM), Vol 3(2), 2014.

[6] G. Porter and G. Doran, "An Anatomical and Photographic Technique for Forensic Facial Identification," Forensic Science International, Vol. 114, pp. 97-105, 2000.

[7] Kevin J. Chan, Stephen J. Elliott, "Mugshot Compliance for Face Image Quality, International Conference on Computational Science and Computational Intelligence, IEEE, pp. 428-432, 2015.

[8] Certified Biometric Professional-Module 1, 2012

[9] Thirimachos Bourlai, Arun Ross, Anil Jain, On Matching Digital Face Images Against Scanned Passport Photos, IEEE International Conference on Biometrics, Identity and Security, 2009.

[10] Anil K. Jain, Brendan Klare, Usang Park, Face Recognition: Some Challenges in Forensics, $9^{\text {th }}$ IEEE International Conference on Automatic Face and Gesture Recognition, 2011.

[11] Rabia Jafri, Hamid R. Arabnia, A Survey of Face Recognition Techniques, Journal of Information Processing Systems, Vol. 5(2), pp. 41-68, 2009.
[12] Anil K. Jain, Brendan Klare, Unsang Park, Face Matching and Retrieval in Forensics Applications, Multimedia in Forensics, Security and Intelligence, pp. 20-28, 2012.

[13] Mian, A.S, Bennamoun, M. \& Owens, R., An Efficient Multimodal 2D3D Hybrid Approach to Automatic Face Recognition, IEEE Transaction on Pattern Analysis and Machine Intelligence 29(11), pp.1927-1943, 2007.

[14] Kusuma,G. P. \& Chua, C.-S., PCA-based image recombination for multimodal 2D+3D face recognition. Image and Vision Computing 29 (5), pp. 306-316, 2011.

[15] Chang, K., Bowyer, K., \& Flynn, P. 2003. Multi-modal 2d and 3d Biometrics for Face Recognition.Proc. of the IEEE International Workshop on Analysis and Modeling of Faces and Gestures. France, October, pp. 187-195, 2003.

[16] Conde, C., A. Serrano,\& E. Cabello, MULTiMODAL 2D, 2.5D \& 3D FACE VERIFICATION. International Conference on Image Processing, Oct, Atlanta, GA , pp. 2061 - 206, 2006.

[17] Charoenpong, T., Tokai S. \&Hase. H., Facial Expression Recognition from 2.5D Partial Face Data by Using Face Plane.ECTI Transactions on Electrical Eng., Electronics, and Communications 8(1), pp. 32-42, 2010.

[18] Liu, P., Woo, W.L. \&Dlay, S.S., One Colored Image Based 2.5d Human Face Reconstruction, 17th European Signal Processing Conference (EUSIPCO), Glasgow, Scotland, August ,pp. 2584- 2588, 2009.

[19] Lee, H.-S.\& Kim, D., Tensor-Based AAM with Continuous Variation Estimation: Application to Variation-Robust Face Recognition, IEEE Transaction on Pattern Analysis and Machine Intelligence 31(6) pp. 1102 - 1116, 2009.

[20] Baykasoulu, A., Ozbakır, L. \&Tapkan, P. 2007. Artificial Bee Colony Algorithm and Its Application to Generalized Assignment Problem.in Swarm Intelligence: Focus on Ant and Particle Swarm Optimization, F. T. Chan and M. K. Tiwari, Eds. Vienna, Austria: Itech Education and Pub., pp. 113-144

[21] Ma, M., Liang, J., Guo, M., Fan, Y. \& Yin, Y. 2011. SAR Image Segmentation Based on Artificial Bee Colony Algorithm.Applied Soft Computing 11 (8): 5205-5214

[22] Jin, W., Zhang, J.\& Zhang, X. 2011. Face Recognition Method Based on Support Vector Machine and Particle Swarm Optimization. Expert Systems with Applications 38(4): 4390-4393

[23] Nazri A. Zamani, A. D Zaharudin, Siti Norul Huda Sheikh Abdullah, Md Jan Nordin, Sparse representation super-resolution method for enhancement analysis in video forensics, proceeding of 12th International Conference on Intelligent Systems Design and Applications, pp. 921-926, 2012.

[24] Liu, X. 2010.Video-based face model fitting using Adaptive Active Appearance Model.Image and Vision Computing 28: 1162-1172. 